\title{
Extracting 3-D deformation fields from left- and right-looking InSAR with SM-VCE method: a case study of October 21, 2016 Central Tottori earthquake
}

\author{
J. H. Liu ${ }^{1}$, J. Hu$^{1}$, Z.W. Li ${ }^{1}$ \\ ${ }^{1}$ School of Geosciences and Info-Physics, Central South University, Changsha 410083, Hunan, P.R. China - (liujihong, csuhujun, \\ zwli)@csu.edu.cn
}

KEY WORDS: InSAR, 3-D deformations, SM-VCE, left-looking mode, accuracy assessment

\begin{abstract}
:
Three-dimensional (3-D) deformation fields with respect to the October 2016's Central Tottori earthquake are extracted in this paper from ALOS-2 conducted Interferometric Synthetic Aperture Radar (InSAR) observations with four different incline angles, i.e., ascending/descending and left-/right-looking. In particular, the Strain Model and Variance Component Estimation (SM-VCE) method is developed to integrate the heterogeneous InSAR observations without being affected by the coverage inconformity of SAR images associated with the earthquake focal area. Compare with classical weighted least squares (WLS) method, SM-VCE method is capable for the retrieval of more accurate and complete deformation field of Central Tottori earthquake, as indicated by the comparison with the GNSS observations. In addition, accuracies of heterogeneous InSAR observations and 3-D deformations on each point are quantitatively provided by the SM-VCE method.
\end{abstract}

\section{INTRODUCTION}

Differential Interferometric Synthetic Aperture Radar (SAR, InSAR, DInSAR) has been proved a promising geodetic tool, mainly featured by its spatially high density of measurements, large scale of spatial coverage and high measurement accuracy, in monitoring the regional ground deformation associated with geological events such as earthquakes, volcanos, landslides, ice flows and land subsidence (Fialko et al., 2005; Hamling et al., 2017; J. Hu et al., 2014a; Jung et al., 2011). While the onedimensional measurements along the line-of-sight (LOS) can be an obstacle of DInSAR when retrieving the full threedimensional (3-D. i.e., east, north and up components, respectively.) surface deformations with respect to only one pair of SAR images on the study zone. Consequently, multiple rightlooking DInSAR measurements with greatly varied incidence angle are used to generate 3-D surface deformations associated with high-latitude regions (Gray, 2011; Wright et al., 2004), which can derive 3-D deformations with high accuracy but is impotent in mid-, low-latitude region. Moreover, the Pixel Offset-Tracking (POT) and/or Multi-Aperture InSAR (MAI) techniques, featured by ungraded accuracy but providing the complementary azimuth (AZI) direction deformation compare to DInSAR, are introduced to obtain 3-D surface deformations triggered by geophysical dynamics (e.g., earthquakes, volcanos), based on both ascending and descending SAR images (Jung et al., 2011; Michel et al., 1999). Recently, the left-looking mode of SAR satellites is available as well, which is accompanied with notably different geometry in the process of imaging compare to the right-looking mode, making it possible that generating full 3D surface deformation maps, from only ascending and descending DInSAR measurements associated with the study zone (Yu et al., 2016).

An earthquake (Mw6.2) occurred in Central Tottori Prefecture in Japan on October 21, 2016. ALOS-2 conducted SAR satellites have imaged this region before and after the event, and both ascending and descending measurements, with left- and rightlooking mode, are available, indicating it possible to obtain 3-D surface deformations associated with this events. For precise 3D deformations retrieval, a method named as SM-VCE (Strain Model and Variance Component Estimation), which considers both 3-D deformations relationship between adjacent points and weighting factors of each kind of measurements (Liu et al., 2018), is presented.

\section{DATA AND METHODOLOGY}

\subsection{Data}

The event occurred in Central Tottori in Japan on October 21, 2016 is observed by four interferometric pairs acquired from ALOS-2 PALSAR-2 ascending and descending orbits, both with left- and right-looking modes, respectively (i.e., Table 1). The footprints of these four interferograms are shown in Figure 1(a). The study zone (i.e., red rectangular in Figure 1(a)), sorted by footprints of observed data, can be split into three parts, i.e., part $\mathrm{A}$, part $\mathrm{B}$ and part $\mathrm{C}$, respectively, and each part is imaged by various interferograms (e.g., part A is imaged by ascending rightlooking, descending right-looking and descending left-looking mode interferograms). For generating LOS direction measurements, two-pass DInSAR technique is conducted.

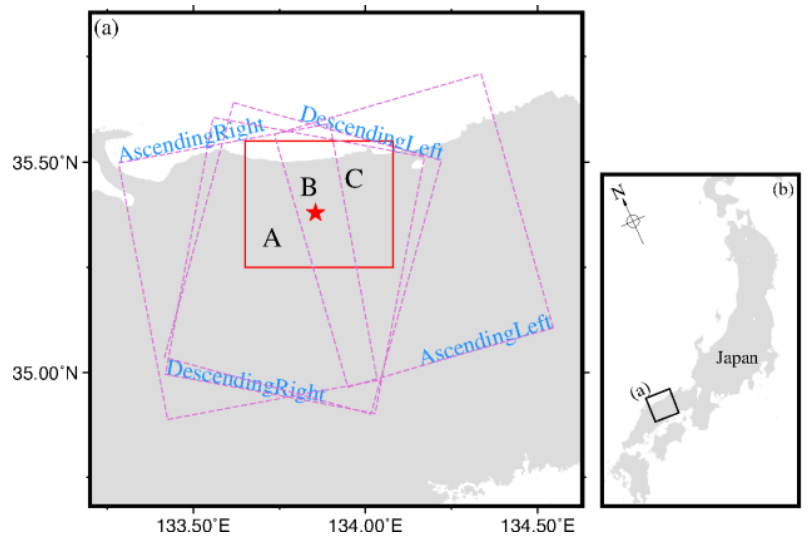

Figure 1. Footprints of the used ALOS-2 PALSAR-2 data. In (a), the red box indicates the limit of study area, the dashed orchid boxes outline the footprints of each interferogram and the red star represents the epicenter of this earthquake. (b) shows the location of the study area relative to Japan

The final geocoded deformation maps in the LOS direction of 
different viewing angles are exhibited in Figure 2. The descending interferometric one with left-looking angle (Figure 2(c)), though spanning the time interval about two years, is still highly coherent in the study area, due to the robust of L-band SAR in terms of temporal decorrelation (Strozzi et al., 2008). As expected, the deformation pattern of interferograms extracted by the ascending left-looking (AsL) and descending right-looking (DesR) measurements, respectively, are very similar, as are the ascending right-looking (AsR) and descending left-looking (DesL) images (Wright et al., 2004). While both of interferograms from the ascending orbit image only part of the study area, inducing unfortunate impact on the performance of recovering 3-D surface deformations in the whole region.

\begin{tabular}{|c|c|c|c|c|}
\hline Paras. & \multicolumn{4}{|c|}{ Pairs } \\
\hline As/Des & As & As & Des & Des \\
L/R & Left & Right & Left & Right \\
Date & $01 / 17 / 15$ & $05 / 23 / 16$ & $12 / 07 / 14$ & $08 / 03 / 16$ \\
& $10 / 22 / 16$ & $10 / 24 / 16$ & $10 / 23 / 16$ & $10 / 26 / 16$ \\
\hline
\end{tabular}

Table 1. Information of the used SAR data
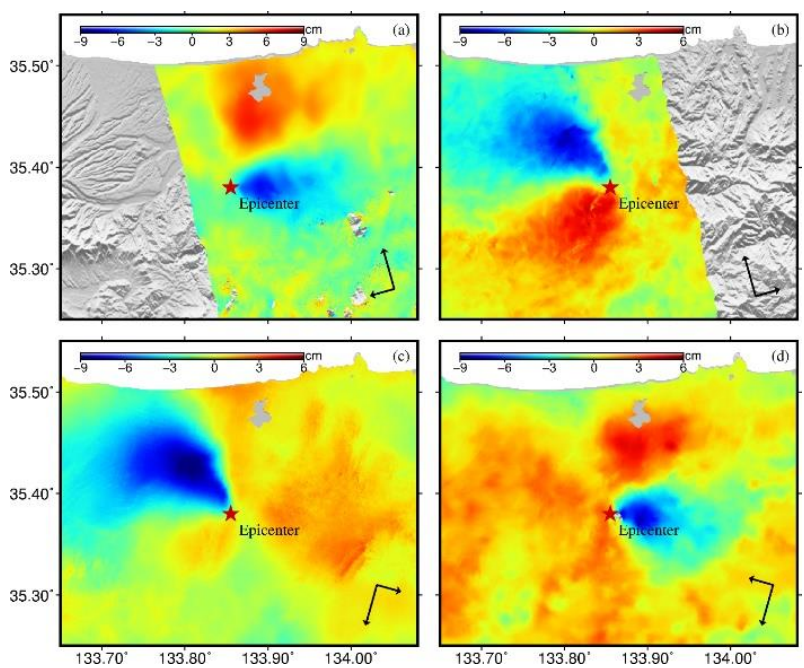

Figure 2. LOS deformation measurements of the central Tottori Earthquake along the (a) ascending left-looking, (b) ascending right-looking, (c) descending left-looking and (d) descending right-looking, respectively. Longer arrows correspond to along track directions and shorter ones indicate the LOS directions from satellites to ground points, respectively

\subsection{Methodology}

To retrieve the full 3-D surface deformations, a method termed as SM-VCE is introduced based on the DInSAR extracted LOS direction measurements. This method is potential to retrieve 3-D deformations with InSAR by integrating the Strain Model (SM) and Variance Component Estimation (VCE) algorithm, which can exploit the spatial correlation of the adjacent points' deformations and produce accurate weights for multiple InSAR measurements (Liu et al., 2017). As far as the procedure of SMVCE is concerned, the observation functions based on SM between the 3-D deformations of the interested point and InSAR measurements should be firstly established, followed by the VCE algorithm for accurate weights determination and Weighted Least Squares (WLS) method for the final solution, which can simultaneously estimate the 3-D deformations as well as SM parameters (see Liu et al. (2017) for details).

\section{RESULTS}

\subsection{Three-dimensional deformations}

A complete 3-D deformations field is derived from only DInSAR measurements with four distinct viewing angles (i.e., AsL, AsR, DesL and DesR) based on the recently proposed innovative method SM-VCE. Figure 3 shows a classical picture of the estimated 3-D deformations associated with the October 2016's Central Tottori earthquake. The maximum deformations in the east, north and up component are about $8.3 \mathrm{~cm}, 18.4 \mathrm{~cm}$ and $5.1 \mathrm{~cm}$, respectively. The study zone can be divided into four sections with respect to the 3-D deformation patterns. In the southeast and northwest sections, an uplift vertical and epicenter-ward horizontal deformation pattern is observed while the opposite deformation pattern appears in the southwest and northeast zones. The GNSS observed results are involved in to assess the accuracy of InSAR derived deformations. The Root Mean Square Errors (RMSEs) are $1.6 \mathrm{~cm}, 3.0 \mathrm{~cm}$ and $0.9 \mathrm{~cm}$ respectively in the east, north and up directions. The most accurate estimation for the up component is expected as the only involved DInSAR measurements are of great sensitivity to the vertical deformation especially with those dataset possessing various diversity of incident angles. The north component, with the highest magnitude of deformation, shows a lowest accuracy compare to the east and up ones.

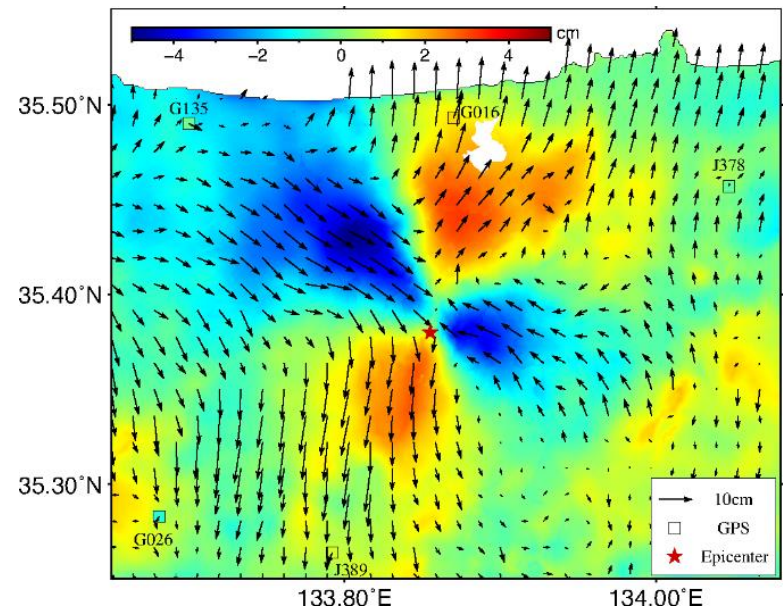

Figure 3. Three-dimensional deformations associated with the

focal zone. The colored base map corresponds to vertical deformation and the arrows show the sub-sampled horizontal deformations. Rectangles show the location of GNSS stations

\subsection{Accuracy assessment}

Integrating SM-VCE method, we can obtain the Standard Deviations (SDs) of each measurement from the VCE determined variance components $\sigma_{i}^{2}$ and weighting factors $P_{i}$

$$
S D_{i}=\frac{\sigma_{i}^{2}}{P_{i}}
$$

Figure 4 shows the histograms of the estimated SDs with respect to the original observations (i.e., AsL, AsR, DesL and DesR). Generally, accuracies of InSAR observations are at the level of several millimetres, and only part of AsL observations shows higher SDs, revealing the capability of InSAR in monitoring deformations. While SDs of AsL observations (Figure 4(a)) shows different distribution pattern where two peaks are appear and the range of SD values are larger than those of others. This can be ascribed to the quite different accuracy between the upper 
and lower part of AsL observations.
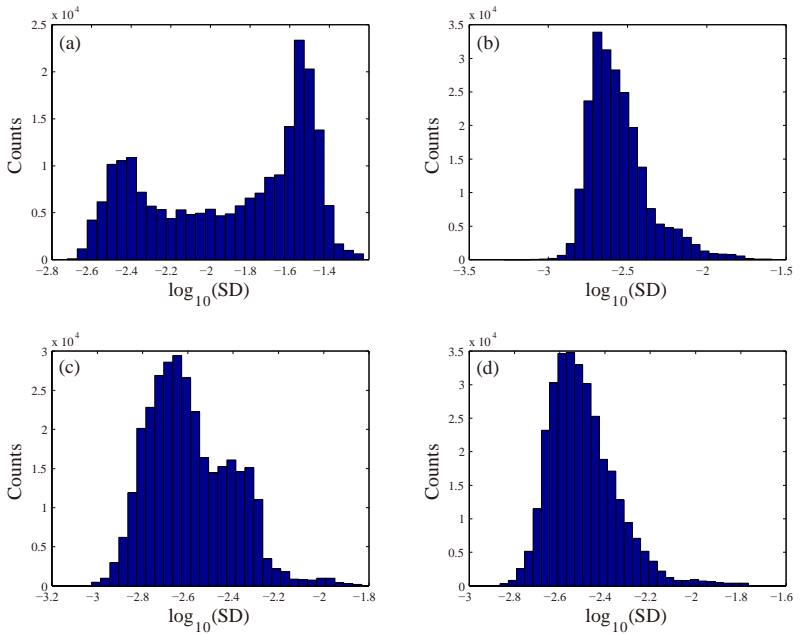

Figure 4. Histograms of the estimated SDs with respect to (a) ascending left-looking, (b) ascending right-looking, (c) descending left-looking and (d) descending right-looking observations, respectively

\section{CONCLUSIONS}

Complete 3-D deformation fields of 2016 Central Tottori earthquake are generated in this paper by exploiting SM-VCE method, based on the introduction of ALOS-2 PALSAR-2 ascending and descending orbits data, both with left- and rightlooking operation modes, respectively. The novel method SMVCE can derive a smoother and more complete deformation field with RMSEs $1.6 \mathrm{~cm}, 3.0 \mathrm{~cm}$ and $0.9 \mathrm{~cm}$ respectively in the east, north and up directions compared with the GNSS observations, yielding this method a potential tool in interpreting and assessing geological hazards. Additionally, the Standard Deviations (SDs) of each measurement can be estimated from the VCE determined variance components and weighting factors, indicating a novel way for the assessment of InSAR measurements.

\section{ACKNOWLEDGEMENTS}

The ALOS-2 PALSAR-2 data were provided by the Japan Aerospace Exploration Agency (JAXA).

\section{REFERENCES}

Fialko, Y., Sandwell, D., Simons, M., et al., 2005, Threedimensional deformation caused by the Bam, Iran, earthquake and the origin of shallow slip deficit, Nature, 435(7040), pp. 295 299.

Gray, L., 2011, Using multiple RADARSAT InSAR pairs to estimate a full three-dimensional solution for glacial ice movement, Geophys. Res. Lett., 38(38), pp. 132-140.

Hamling, I. J., Hreinsdóttir, S., Clark, K., et al., 2017, Complex multifault rupture during the $2016 \mathrm{Mw} 7.8$ Kaikōura earthquake, New Zealand, Science, 356(6334).

Hu, J., Li, Z. W., Ding, X. L., Zhu, J. J., et al., 2014, Resolving three-dimensional surface displacements from InSAR measurements: A review, Earth Sci. Rev., 133(2), pp. 1-17.
Jung, H. S., Lu, Z., Won, J. S., et al., 2011, Mapping ThreeDimensional Surface Deformation by Combining MultipleAperture Interferometry and Conventional Interferometry: Application to the June 2007 Eruption of Kilauea Volcano, Hawaii, IEEE Geosci. Remote Sens. Lett., 8(1), pp. 34-38.

Liu, J. H., Hu, J., Li Z. W., et al., 2018, A Method for Measuring 3-D Surface Deformations With InSAR Based on Strain Model and Variance Component Estimation, IEEE Trans. Geosci. Remote Sens., 56(1), pp. 239-250.

Michel, R., Avouac, J. P., Taboury, J., 1999, Measuring ground displacements from SAR amplitude images: Application to the Landers Earthquake, Geophys. Res. Lett., 26(7), pp. 875-878.

Strozzi, T., Kouraev, A., Wiesmann, A., et al., 2008, Estimation of Arctic glacier motion with satellite L-band SAR data, Remote Sens. Environ., 112(3), pp. 636-645.

Wright, T. J., Parsons, B. E., and Lu Z., 2004, Toward mapping surface deformation in three dimensions using InSAR, Geophys. Res. Lett., 31(1), pp. 169-178.

Yu, M., Kobayashi, T., Yarai, H., 2016, Three-dimensional deformation mapping of a dike intrusion event in Sakurajima in 2015 by exploiting the right- and left-looking ALOS-2 InSAR, Geophys. Res. Lett., 43(9), pp. 4197-4204.

Revised March 2018 